University of New Hampshire

University of New Hampshire Scholars' Repository

Space Science Center

Institute for the Study of Earth, Oceans, and

Space (EOS)

1993

\title{
The design of a gamma-ray burst polarimeter
}

\author{
Mark L. McConnell \\ University of New Hampshire - Main Campus, mark.mcconnell@unh.edu \\ D J. Forrest \\ University of New Hampshire - Main Campus \\ K Levenson \\ University of New Hampshire - Main Campus \\ W T. Vestrand \\ University of New Hampshire - Main Campus
}

Follow this and additional works at: https://scholars.unh.edu/ssc

Part of the Astrophysics and Astronomy Commons

\section{Recommended Citation}

The design of a gamma-ray burst polarimeter McConnell, M. and Forrest, D. and Levenson, $\mathrm{K}$. and Vestrand, W. T., AIP Conference Proceedings, 280, 1142-1146 (1993), DOI:http://dx.doi.org/10.1063/ 1.44145

This Conference Proceeding is brought to you for free and open access by the Institute for the Study of Earth, Oceans, and Space (EOS) at University of New Hampshire Scholars' Repository. It has been accepted for inclusion in Space Science Center by an authorized administrator of University of New Hampshire Scholars' Repository. For more information, please contact Scholarly.Communication@unh.edu. 


\section{AIP $\mid$ Proceedings}

\section{The design of a gammaray burst polarimeter}

M. McConnell, D. Forrest, K. Levenson, and W. T. Vestrand

Citation: AIP Conference Proceedings 280, 1142 (1993); doi: 10.1063/1.44145

View online: http://dx.doi.org/10.1063/1.44145

View Table of Contents:

http://scitation.aip.org/content/aip/proceeding/aipcp/280?ver=pdfcov

Published by the AIP Publishing

\section{Articles you may be interested in}

The spectroscopy of classical gammaray bursts

AIP Conf. Proc. 366, 206 (1996); 10.1063/1.50248

Exploration of bimodality in gammaray burst duration and hardness distributions AIP Conf. Proc. 307, 172 (1994); 10.1063/1.45913

DMSP satellites as gammaray burst detectors

AIP Conf. Proc. 307, 34 (1994); 10.1063/1.45893

The color of gammaray bursts

AIP Conf. Proc. 307, 323 (1994); 10.1063/1.45887

Spectral variations in gammaray bursts

AIP Conf. Proc. 265, 195 (1991); 10.1063/1.42823 
M. McConnell, D. Forrest, K. Levenson and W.T. Vestrand Space Science Center, University of New Hampshire, Durham, NH

\section{ABSTRACT}

The study of the polarization properties of the gamma-ray bursts is the one remaining unexplored avenue of research which may help to answer some of the fundamental problems regarding the nature of these mysterious objects. We have designed an instrument to measure linear polarization in cosmic gamma-ray bursts at energies $>50 \mathrm{keV}$. Here we describe the design of this instrument, which we call the Gamma-RAy burst Polarimeter Experiment (GRAPE).

\section{INTRODUCTION}

The nature of the gamma-ray burst sources has remained a mystery ever since their discovery some 25 years ago. Even after the best efforts of scores of researchers, there is no consensus regarding their origin. The latest results from the Compton GammaRay Observatory (CGRO) have served to heighten the interest in this problem without offering any fundamental answers as to their origin.

When it comes to the study of electromagnetic radiation, there are four measurements which an observer can make (all as a function of time). In the context of gamma-ray burst studies, these are: 1) the source location; 2) the source spectrum (distribution of photon energies); 3) the source intensity (spectrum normalization); and 4) the source polarization. All studies of gamma-ray bursts to date have involved the first three types of measurements. Since these data have not yet yielded any clear fundamental answers as to the nature of the bursts, we contend that an additional piece of information may be required. The only additional piece of information which is available to the observer is that of the polarization of the radiation.

A few authors have discussed polarization effects in the context of burst models. For example, Mitrofanov and Pozanenkol have discussed the polarization inherent in cyclotron emission in a strong magnetic field; they predict a polarization fraction of $\sim 80 \%$ near 100 $\mathrm{keV}$ for a typical neutron star environment. Bisnovatyi-Kogan ${ }^{2}$ has noted that the measurement of polarization in the observed spectral lines would help to determine the exact nature of the line emission. Baring ${ }^{3}$ has examined the polarization produced via magnetic photon splitting; a maximum polarization fraction of about $43 \%$ is possible with this mechanism.

At present, there is no gamma-ray experiment that we know of which is presently in operation or in the planning stages whose major emphasis is the measurement of polarization. The COMPTEL instrument 
on GRO ${ }^{4}$, for example, has only marginal capabilities for detecting polarized emission due to an inefficient geometry (the major drawback for measuring polarization with traditional Compton telescopes). Some of the proposed concepts (such as TTSS ${ }^{5}$ ) include the capability for polarization measurements, but once again, they are not optimized for that purpose.

The prototype GRAPE design has been developed within the context of a long-duration balloon platform. However, the modular nature of this experiment will also facilitate its use as an add-on experiment for satellite platforms. In addition, the simplicity of this design, along with its minimal pointing and service requirements, would make such an experiment ideally suited for deployment on space station Freedom.

\section{POLARIMETER BASICS}

The measurement of linear polarization at hard $x$-ray and gammaray energies is based on the fact that the Compton scattering crosssection is a function of angle from the electric field vector. In particular, the scattering cross-section is a maximum for photons scattered perpendicular to the electric field vector and it is a minimum for photons scattered parallel to the electric field vector. The ratio between the minimum and the maximum scattering cross sections gives a good indication of how effective this approach may be. Although this ratio is quite high $(\sim 65)$ at $100 \mathrm{keV}$, the ratio decreases rapidly at energies above $500 \mathrm{keV}(\sim 5$ at $500 \mathrm{keV})$. This makes this technique increasingly difficult at MeV energies.

\section{THE POLARIMETER MODULE}

GRAPE is designed as a modular experiment, consisting of a number of independent polarimeters. The basic principle of the GRAPE design is to scatter photons from a low-z plastic scintillator into a high-z NaI(T1) scintillator which is capable of absorbing the remaining photon energy with a high probability. A single polarimeter module consists of a ring of individual plastic scintillators surrounding the NaI(Tl) absorber, as shown in Figure 1. The NaI(TI) detector is cylindrical, $7.6 \mathrm{~cm}$ in diameter and $7.6 \mathrm{~cm}$ long. Each plastic scattering element (in the present design) is 7.6 $\mathrm{cm}$ long and $3 \mathrm{~cm}$ thick. A complete module measures $25 \mathrm{~cm} \times 25 \mathrm{~cm}$ in size.

The goal is to measure the azimuthal distribution of the photons scattered into the central detector. This distribution is determined by recording the coincidence events between each of the 12 scattering elements and the central detector. This provides a set of 12 energy-loss spectra which can be used to measure the polarization of the incident flux as a function of energy. 


\section{The Design of a Gamma-Ray Burst Polarimeter}

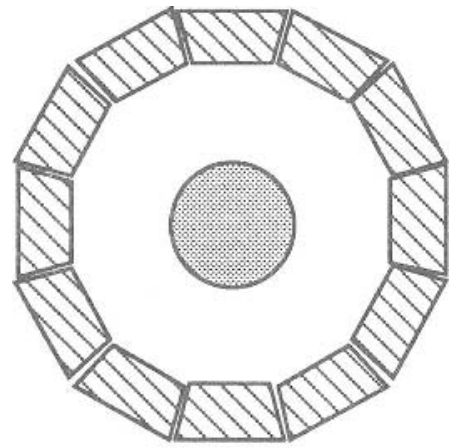

plastic

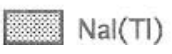

Figure 1. Schematic diagram of a single GRAPE module showing the ring of plastic scintillators which act to scatter incident photons into the central NaI(Tl) detector. In the baseline design, the NaI(Tl) detector is $7.6 \mathrm{~cm}$ in dianeter by $7.6 \mathrm{~cm}$ long. The plastic scattering elements are $3 \mathrm{~cm}$ thick. The plastic detectors are centered on a radius of $11.3 \mathrm{~cm}$. factor,

We define the measured polarization in terms of the modulation

$$
P\left(E_{\gamma}, \theta\right)=\frac{S_{\max }\left(E_{\gamma}, \theta\right)-S_{\min }\left(E_{\gamma}, \theta\right)}{S_{\max }\left(E_{\gamma}, \theta\right)+S_{\min }\left(E_{\gamma}, \theta\right)}
$$

where $S$ refers to the effective area of a single NaI(T1)-plastic scattering pair at incident energy $E_{\gamma}$ and incident angle $\theta$. In this expression, the maximium and minimum values refer to the maximum and minimum of the azimuthal variations. The detection properties inherent in this expression have been studied in detail with a series of Monte Carlo simulations. These simulations employed a modified version of the CERN GEANT code (modified to properly handle polarized photons).

The results of these simulations are shown in Figures 2 and 3 . Figure 2 shows the effect of increasing incident energy on the measured azimuthal variations (for an incidence angle of $0^{\circ}$ ). Figure 3 shows the effect of increasing the incidence angle (for an incident energy of $100 \mathrm{keV}$ ). In all cases, the response both to $100 \%$ linearly polarized radiation and to unpolarized radiation is shown. Solid

\begin{tabular}{|c|c|c|c|c|}
\hline $\begin{array}{c}\text { Energy } \\
(\mathrm{keV})\end{array}$ & $\begin{array}{c}\mathrm{S}_{\mathrm{T}}(\mathrm{NaI}) \\
\mathrm{cm}^{2}\end{array}$ & $\begin{array}{c}\mathrm{S}_{\mathrm{T}} \text { (plast) } \\
\mathrm{cm}^{2}\end{array}$ & $\begin{array}{c}S_{T}(\operatorname{coinc}) \\
\mathrm{cm}^{2}\end{array}$ & $P$ \\
\hline 50 & 6.5 & 153 & 4.1 & $71 \frac{8}{6}$ \\
\hline 100 & 6.4 & 146 & 4.2 & $71 \%$ \\
\hline 200 & 10.2 & 133 & 3.6 & $73 \%$ \\
\hline 300 & 23.8 & 125 & 3.1 & $64 \%$ \\
\hline 400 & 31.6 & 117 & 2.9 & $63 \%$ \\
\hline 500 & 35.3 & 111 & 2.6 & $59 \%$ \\
\hline 700 & 36.9 & 101 & 2.3 & $47 \%$ \\
\hline 900 & 35.6 & 93 & 1.8 & $33 \%$ \\
\hline
\end{tabular}

lines represent a fit to the data. Some of these results are also given in Table I (where $S_{T}$ refers to the summed total of all elements in a single module). We conclude from these simulations that: 1) there is significant efficiency at energies up to 500 
$\mathrm{keV}$; and 2) there is a clear polarization signal even at $30^{\circ}$ offaxis, thus providing for an effective FOV of $\sim 1$ steradian.
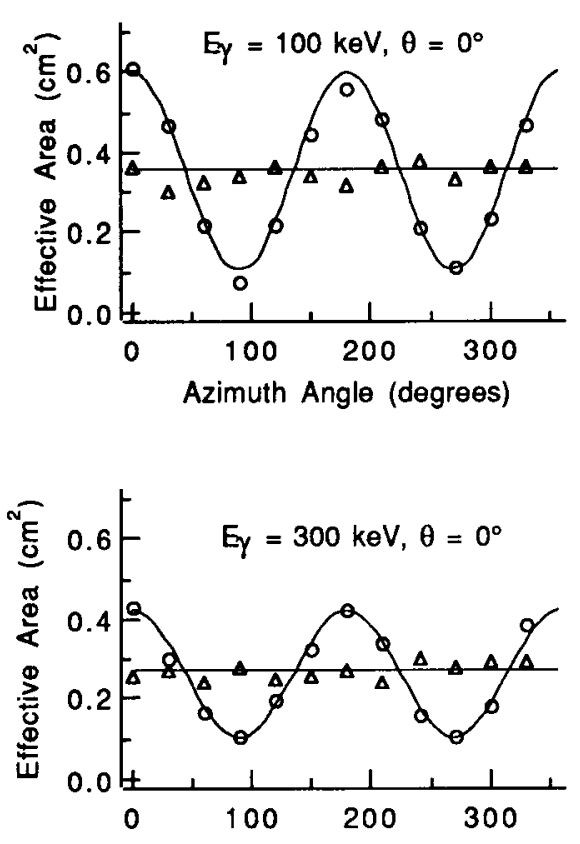

Azimuth Angle (degrees)

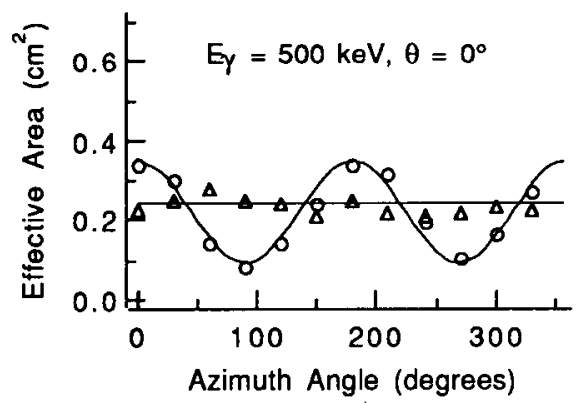

Figure 2. The modulation as a function of incident energy expressed as effective area per scattering pair. In each case, the response to both $100 \%$ polarized flux (circles) and unpolarized flux (triangles) is shown. Errors are roughly the size of the plot symbols.
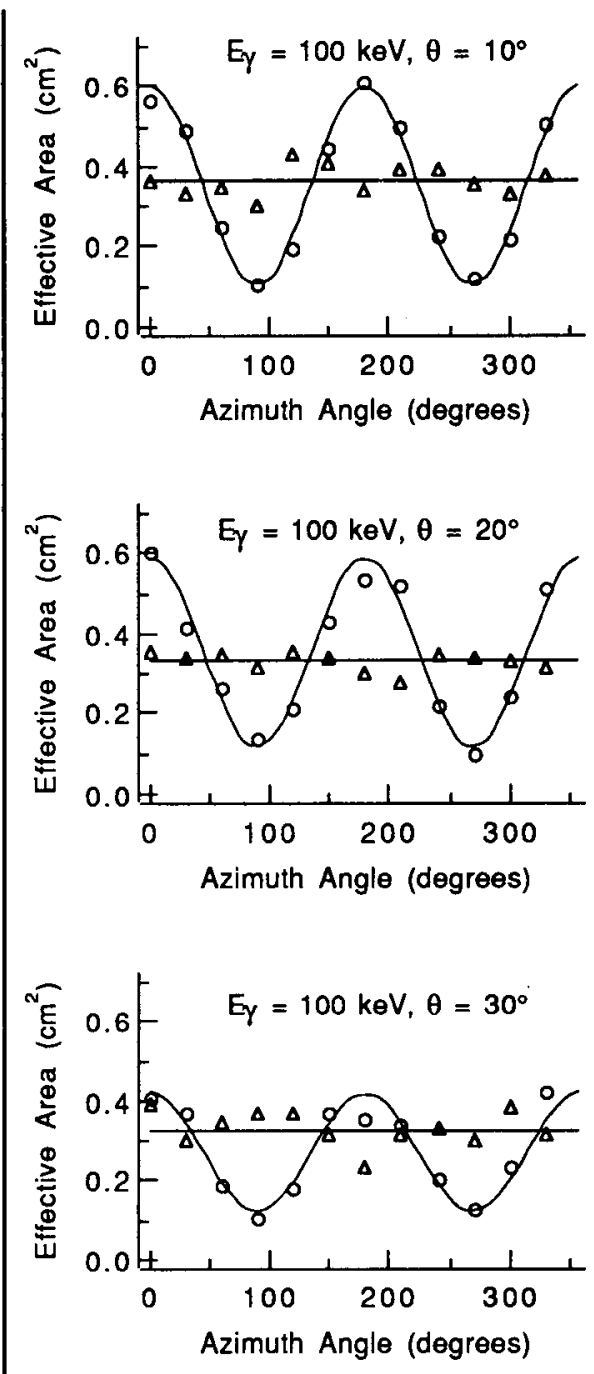

Figure 3. The modulation as a function of incident angle expressed as effective area per scattering pair. In each case, the response to both $100 \%$ polarized flux (circles) and unpolarized flux (triangles) is shown. Errors are roughly the size of the plot symbols. 


\section{The Design of a Gamma-Ray Burst Polarimeter}

\section{BALLOON PAYLOAD CONCEPT}

In developing this concept for a balloon payload, it is necessary for the detector to have an efficiency which would give useful scientific results within the time frame of a typical longduration balloon flight (typically, 2-3 weeks). This requires an array of polarimeter modules. Our baseline design is an array of 16 modules, as shown in Figure 4. As a polarimeter, GRAPE would be able
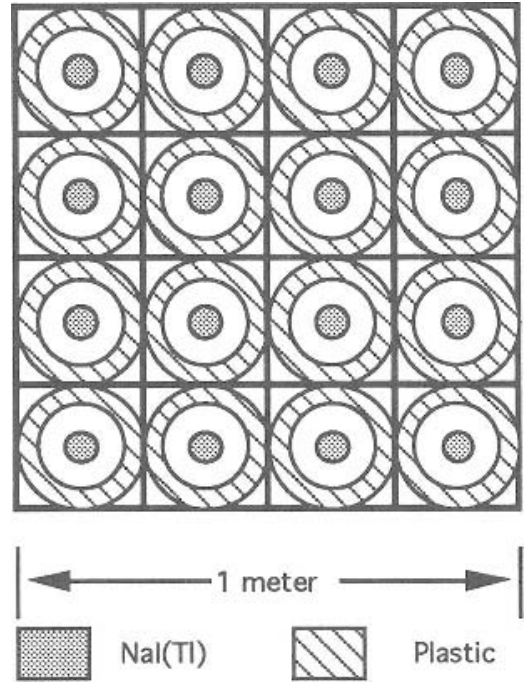

Figure 4. A schematic of the full balloon prototype, consisting of an array of 16 individual modules.

to detect $50 \%$ linear

polarization at $>3 \sigma$ in a burst with a fluence of $5 \times 10^{-6}$ ergs $\mathrm{cm}^{-2}$. (More recent simulations appear to suggest that refinements to this design may improve the detection efficiency by as much as 50\%.) With a FOV of $\sim 1$ steradian, we would expect to see $\sim 1$ burst at this fluence level during a 2-3 week balloon flight. Although GRAPE is optimized for the measurement of polarization (up to several hundred $\mathrm{keV}$ ), the use of the individual detectors will also permit a significant capability for timing, and spectral measurements of gammaray bursts up to several Mev. The use of corner-mounted $x$-ray detectors (as in the BATSE design) will permit localization of bursts to within several degrees; this would be required for a more accurate deconvolution of the GRAPE response (due to its dependence on incidence angle). Finally, systematic effects within the modules would be alleviated by rotation of the balloon payload.

\section{REFERENCES}

1. I. Mitrofanov and A. Pozanenko, in Gamma-Ray Bursts: Observations, Analysis and Theories, eds. C. Ho, R. Epstein, and E. Fenimore (Cambridge Univ. Press, 1992), p. 430.

2. G.S. Bisnovatyi-Kogan, in Gamma-Ray Bursts: Observations, Analysis and Theories, eds. C. Ho, R. Epstein, and E. Fenimore (Cambridge Univ. Press, 1992), p. 89.

3. M.G. Baring, in Compton Observatory Science Workshop, eds. C. Shrader, B. Dennis, and N. Gehrels (NASA CP 3137, 1992), p. 293.

4. V. Schönfelder et al., submitted to Ap. J. Suppl. (1992)

5. K. Hurley, in Gamma-Ray Bursts: Observations, Analysis and Theories, eds. C. Ho, R. Epstein, and E. Fenimore (Cambridge Univ. Press, 1992), p. 279. 